\title{
Undesirable Postoperative Anesthesia Outcomes at Two National Referral Hospitals: A Cross-Sectional Study in Eritrea
}

\author{
Yonatan Mehari Andemeskel iD, 1 Traudl Elsholz, ${ }^{1}$ Ghidey Gebreyohannes, ${ }^{2}$ \\ and Eyasu H. Tesfamariam ${ }^{3}$ \\ ${ }^{1}$ Department of Anesthesia and Critical Care, School of Nursing, Asmara College of Health Sciences, Asmara, Eritrea \\ ${ }^{2}$ Asmara College of Health Sciences, Asmara, Eritrea \\ ${ }^{3}$ Department of Epidemiology and Biostatistics, School of Public Health, Asmara College of Health Sciences, Asmara, Eritrea \\ Correspondence should be addressed to Yonatan Mehari Andemeskel; yonimer2@gmail.com
}

Received 7 May 2020; Revised 17 July 2020; Accepted 3 September 2020; Published 15 September 2020

Academic Editor: Basavana B. Goudra

Copyright (c) 2020 Yonatan Mehari Andemeskel et al. This is an open access article distributed under the Creative Commons Attribution License, which permits unrestricted use, distribution, and reproduction in any medium, provided the original work is properly cited.

\begin{abstract}
Background. Postoperative undesirable anesthesia outcomes are common among patients undergoing surgery. They may affect body systems and lead into more serious postoperative problems. This research is conducted in the Eritrean National Referral Hospitals with the aim of assessing the prevalence of undesirable anesthesia outcomes during the postoperative period. Method. A cross-sectional study design was applied on 470 patients who underwent different types of surgeries within a three-month period. Patients were interviewed 24 hours after operation (POD 1) using the Leiden Perioperative care Patient Satisfaction questionnaire (LPPSq). This study reports one component of a large study conducted. The dimension "Discomfort and needs" of the LPPSq was considered, and the measurements of that dimension are presented in this report. Items of the dimension were standardized and measured using a five-point Likert scale from "Not at all" to "Extremely." Multivariable logistic regression was used to look for the association of the outcomes with the types of surgery and types of anesthesia using SPSS (Version 22). Results. The prevalence were computed in two manners, prevalence of those with 'at least a little bit' outcomes, which was computed to see the total occurrence of these outcomes, and prevalence of those having 'more than moderate' outcomes to see the severe experience of these outcomes. Prevalence of the predominant undesirable outcome, postoperative pain, for 'at least a little bit' and 'more than moderate' were $82.6 \%$ and $43.6 \%$, respectively. The rest of the postoperative undesirable outcomes were less frequently reported. Conclusion. Postoperative pain was found to be the most prevalent undesirable outcome. Enhancement of proper assessment and management of postoperative pain through the development and implementation of specific pain management modalities is needed.
\end{abstract}

\section{Background}

Undesirable postoperative anesthesia symptoms are common and may affect all patient's body systems [1]. The most commonly mentioned complications are pain, nausea, vomiting, sore throat, shivering, thirst, and hunger [1-3]. These complications has also been observed to trouble patients from the Eritrean National Referral Hospitals during their postoperative stay. Underestimation of these undesirable events and lack of adequate management protocols and skills persist to enhance their occurrence.

Postoperative pain is a common experience for postoperative patients and remains a serious problem facing anesthesia providers in their daily practice [4]. Poor management of acute postoperative pain is among the causes for some medical complications, and it prolongs the time of recovery and hospitalization increasing postoperative morbidity $[5,6]$. Although the authors could not find any study to demonstrate the quality of postoperative pain management at the level of patients, pain is undervalued, and insufficient knowledge of pain management persists. As an acute care management, diclofenac as an intramuscular injection is the commonly prescribed drug followed by the orally taken NSAIDs. Opioids are hardly utilized, associated with the lack of knowledge, general fear of utilizing opioids, and most importantly shortage of availability. Moreover, 
postoperative pain medications are still prescribed on as needed basis requiring patients to ask for pain medication, and treatment is usually provided when patients experience severe pain. Meanwhile, analgesia should be the fundamental right of every patient, and allowing patients to experience postoperative pain is unacceptable and unethical especially when tools and educated health care providers are available [7]. Proper postoperative pain management significantly enhances recovery and reduces patient morbidity increasing the hospital stay and cost $[6,8]$. Thorough evaluation and objective measuring of pain with the pain measuring scales such as the visual analog scale is important as it helps in determining the effectiveness of treatment [9-11]. Postoperative nausea, vomiting, and hypothermia are also among the frequently occurring undesirable events $[12,13]$. Generally, failing to prevent these undesirable events have been linked to subsequent, more serious postoperative problems [2]. They have also been explained as modifiable sources of patient dissatisfaction [3].

The effectiveness of managing these undesirable events is determined by the correct and timely detection of the symptoms and applying appropriate pharmacotherapy [1]. Recognizing and treating these complications is vital in the provision of good quality of care $[1,2]$. Meanwhile, it has been observed in the study settings that these undesirable postoperative events to be common troubling postoperative patients, increasing their morbidity and prolonging their recovery and hospital stay. No local guideline or management protocol exists for the management of these undesirable events. A patient who undergoes anesthesia is at risk of complications, and the anesthetist is expected to be responsible in securing patient safety through adequate management of these risks and outcomes $[14,15]$. Meanwhile, in Eritrea, the work of anesthetists is usually confined only to the operating room. No anesthetist is involved in the management of patients during their postoperative stay unless the patient undergoes in to an unexpected condition that requires emergency management. Hence, meaningful anesthetic evaluations and management of postoperative patient outcomes are hardly a practice in these settings. This study, the first of its kind in the country, is therefore aimed at determining the prevalence of anesthesia-related postoperative undesirable outcomes among patients undergoing surgery in the selected hospital settings. The results could reliably show areas of intervention to improve the quality management of the postoperative undesirable events be discussed, and effective management protocols could be formulated consistent with the international guideline that would optimize the management of these undesirable events so as to improve the quality of care. The results will also extend the body of literature and serve as a baseline for further studies.

\section{Methods}

2.1. Study Design and Setting. This descriptive, cross-sectional study was conducted between January and March of 2018 in Eritrea, a country in the horn of Africa. Eritrea has two National Referral Hospitals, which are located at the
Capital city, Asmara. They are called Halibet and Orotta National Referral Hospitals and both of them provide health services at a tertiary level. They are the only governmental medical surgical national referral hospitals in which all types of major and minor surgeries take place.

2.2. Participants. During the study period, a total of 526 patients underwent surgeries under general and regional anesthesia. Respondents' eligibility was based on their willingness to participate in the study. Patients under the age of 18 years, those who were discharged before 24 hours of postoperative period, patients with serious illness, and those who did not consent to participate were excluded from the study, and the final sample size was 470 .

2.3. Data Collection Tool and Method. The key elements of sociodemographic and clinical characteristics of the patients were obtained using a sociodemographic and clinical form. The sociodemographic and clinical details obtained were age, gender, place of residence (urban or rural), occupation, hospital setting, health coverage, type of anesthesia, type of surgery, and admission type (emergency or elective). The undesirable anesthesia outcomes were measured using the dimension "discomfort and needs" of the Leiden Perioperative care Patient Satisfaction questionnaire (LPPSq). The LPPSq was initially modified by Calijouw et al., and permission was asked and obtained from the responsible author. The LPPSq is a validated suitable research scale [16], having six dimensions, in which 'discomfort and needs' was separately handled and analyzed because of its unique psychometric characteristics $[17,18]$. As far as the founders of the scale are concerned, the internal consistency within the discomfort and need dimension was so low that it was not incorporated to get a composite score of the LPPSq, resulting to analysis of the individual items separately. This of course was one of the clear indications that the items listed in the dimension provide wider objectives and another perspective of anesthesia service. Explanation of the five other dimensions is made in previous publication [19]. The 'discomfort and needs' investigates the common adverse outcomes of anesthesia raised from patient's perspective including postoperative pain, sore throat, back pain, nausea, vomiting, cold, hunger, thirst, and headache.

The study was conducted by independent researches who were not involved in any of the perioperative anesthetic or any other postoperative management of patients in the study settings, and data were collected through face to face interview by four well trained anesthetists who do not work in the study settings. They were also assistant researches and were well aware of the study objectives.

2.4. Data Collection Procedure. The researchers visited each hospital and explained the purpose of the study and its clinical significance to the hospital directors after getting the ethical clearance approval by the Research and Human Resources Development, Ministry of Health. Permission to conduct the study was then obtained from each hospital 
director. Recruitment of the patients was undergone before the moment of their operation. After full explanation of the study objectives and assurance of confidentiality and anonymity, patients were given written informed consent. The interview was then conducted in their respected postoperative wards after assuring that they felt comfortable for the interview, and the time to complete the questionnaire was about 15-20 minutes.

2.5. Variables and Measures. The items in the 'discomfort and need' were standardized and measured using a fivepoint Likert scale. Patients had to state to which degree they experienced each of the attribute stated in each item after operation. The replies to the items were "Not at all" (0), "A little bit" (1), "Moderately" (2), "Quite a bit" (3), and "Extremely" (4). The items were then computed into two sequences of prevalence, prevalence of those with 'at least a little bit' undesirable anesthesia outcome occurrence, which was computed to see the total occurrence of these undesirable outcomes, and prevalence of those having 'more than moderate' postoperative undesirable anesthesia outcome occurrences to see the severe occurrence of these outcomes.

2.6. Validity and Reliability. Content validity of the items of the dimension was checked along with the rest of the dimensions by an expert' opinion from the anesthesia department. Translation (to the local language) was done by a bilingual language expert and was then back translated to English by another bilingual person who was not aware of the study objectives. A pretest was also performed to ascertain the comprehension and understandability of the questions. Internal consistency of the dimension was also checked (Chronbach $\alpha=0.66$ ).

2.7. Data Analysis. Responses were coded and entered into SPSS (Version 22) statistical software for analysis. Data cleaning and preliminary explorations were performed to assure accuracy of entry before conducting the main analysis. Frequency (percentage) and mean (standard deviation) were used to summarize the demographic and clinical variables of the participants. The prevalence of those with 'at least a little bit' outcome occurrence and prevalence of those with 'more than moderate occurrence' were also computed. Moreover, odds ratios (95\% CI) were computed to assess the association of undesirable anesthesia outcomes with the types of surgery and types of anesthesia using multivariable logistic regression. $P$ values less than 0.05 were considered as significant throughout the analyses.

\section{Results}

3.1. Population Characteristics. A total of 526 patients underwent surgeries under general and regional anesthesia out of which 470 fulfilled the inclusion criteria and were included in data analysis. Figure 1 shows a summary of the ultimate number of participants and their eligibility.

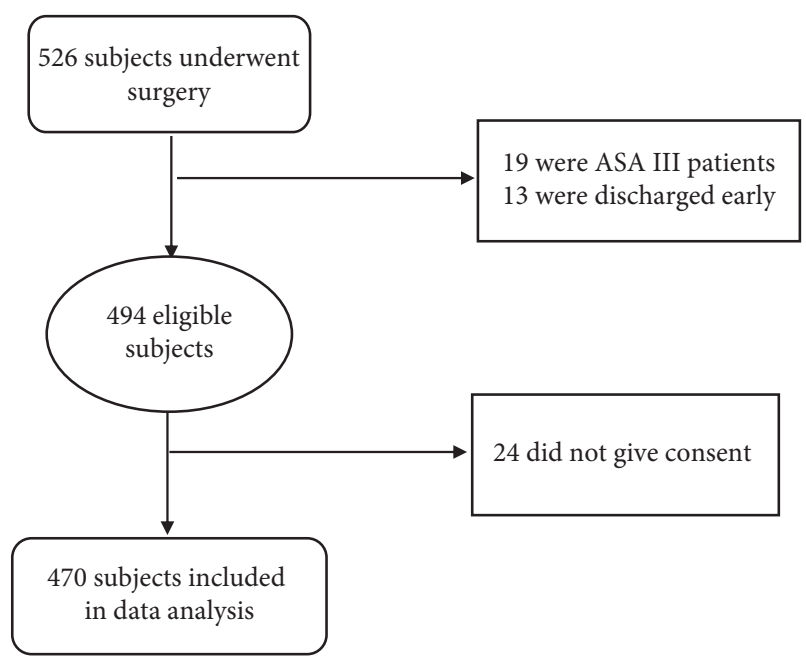

FIGURE 1: Eligible number of patients for the study.

The demographic and clinical details of the participants are shown in Table 1. The age of the respondents ranged from 18 to 85 years, with a mean \pm SD value of $45.9 \pm 14.7$. From the total 470 participants, $55.1 \%$ were males and $44.9 \%$ were females. The majority (63.2\%) of the patients were from Orotta Hospital. The patients underwent a wide range of surgical procedures, including general, orthopedic, Gyn/obs, ENT, and burn surgery. 267 (56.8\%) patients had general anesthesia and 203 (43.2\%) had regional anesthesia.

\subsection{Undesirable Postoperative Anesthesia Outcomes.} Table 2 shows the prevalence with 'at least a little bit' and 'more than moderate' undesirable anesthesia outcomes. The predominant undesirable anesthesia outcome was postoperative pain, followed by cold, nausea, vomiting, thirst, back pain, headache, sore throat, and hunger. The prevalence of 'at least a little bit' and 'more than moderate' postoperative pain were $82.6 \%$ and $43.6 \%$, respectively.

3.3. Association of Outcomes with Types of Surgery and Types of Anesthesia. As shown in Table 3, the association of the postoperative undesirable anesthesia outcomes with the types of surgery the patients underwent and the types of anesthesia those patients took were computed.

Significant difference in the occurrence of nausea and vomiting occurred in those patients who did general surgery. The odds of nausea $(\mathrm{OR}=1.74,95 \% \mathrm{CI}: 1.04,2.90)$ and vomiting $(\mathrm{OR}=2.03,95 \% \mathrm{CI}: 1.19,3.47)$ were higher among patients who took general anesthesia as compared to those who took regional anesthesia. On the other hand, significant experience of back pain was found in those patients who took regional anesthesia with the scores of $51 \%(\mathrm{OR}=0.49,95 \%$ CI: $0.27,0.88)$ in those who did general surgeries and $67 \%(\mathrm{OR}=0.33,95 \% \mathrm{CI}$ : 0.14 , 0.80 ) in those who did Gyn/obs surgeries.

\section{Discussion}

This is the first survey in Eritrea that discusses about undesirable postoperative anesthesia-related events. It focuses 
Table 1: Demographic and clinical characteristics of the participants.

\begin{tabular}{lccc}
\hline Variables & & Frequency & Percentage \\
\hline \multirow{2}{*}{ Gender } & Male & 259 & 55.1 \\
& Female & 211 & 44.9 \\
\hline \multirow{2}{*}{ Residence } & Urban & 274 & 58.3 \\
& Rural & 196 & 41.7 \\
\hline \multirow{2}{*}{ Occupation } & Employed & 235 & 50 \\
& Unemployed & 235 & 50 \\
\hline \multirow{2}{*}{ Hospital setting } & Halibet & 173 & 36.8 \\
& Orotta & 297 & 63.2 \\
\hline \multirow{2}{*}{ Health coverage } & Paying & 358 & 76.2 \\
& Free & 112 & 23.8 \\
\hline \multirow{2}{*}{ Type of anesthesia } & General & 267 & 56.8 \\
& Regional & 203 & 43.2 \\
\hline \multirow{4}{*}{ Type of surgery } & General & 261 & 55.5 \\
& Orthopedic & 99 & 21.1 \\
& Gyn/obs & 89 & 18.9 \\
& ENT & 7 & 1.5 \\
\multirow{2}{*}{ Admission type } & Burn & 14 & 3.0 \\
\hline \multirow{2}{*}{ Age } & Emergency & 109 & 23.2 \\
& Elective & 361 & 76.8 \\
\hline \multirow{2}{*}{} & Mean & SD & \\
& 45.87 & 18.53 & \\
\hline
\end{tabular}

TABLE 2: Prevalence of undesirable postoperative anesthesia outcomes $(n=470)$.

Prevalence of UAO

Discomfort and needs At least a little bit More than moderate

\begin{tabular}{lcc} 
& $n(\%)$ & $n(\%)$ \\
\hline Postoperative pain & $388(82.6)$ & $205(43.6)$ \\
Sore throat & $87(18.5)$ & $10(2.1)$ \\
Back pain & $125(26.6)$ & $20(4.3)$ \\
Nausea & $191(40.6)$ & $44(9.4)$ \\
Vomiting & $177(37.7)$ & $47(10)$ \\
Cold & $194(41.3)$ & $80(17)$ \\
Hunger & $86(18.3)$ & $21(4.5)$ \\
Thirst & $130(27.7)$ & $47(10)$ \\
Headache & $118(25.1)$ & $25(5.3)$ \\
\hline
\end{tabular}

Severity was graded as $0=$ not at all; $1=$ a little bit; $2=$ moderately; $3=$ quite a bit; $4=$ extremely.

on the most common undesirable postoperative anesthesia outcomes raised from patients' perspectives. One thing that should be considered is that the findings should be interpreted and understood within the context of the barriers of pain management being a developing country in which there is no national guideline that exist for the management of pain.

Generally, the management of these events is usually underestimated and is suboptimal, especially when it comes to the management of postoperative pain. The involvement of the anesthetist is very important in the management of these events. Moreover, having a local standard guideline is an important strategy in promoting standardization of procedures and patient controlled management. However, these two factors are not yet in practice in the study settings.
The work of the anesthetist does not go beyond the operating room, and the management of these events is left either to the surgeon responsible or to the postoperative nurses. Moreover, unstandardized management protocol, shortage of experts in pain management, lack of adequate pain medications, and lack of knowledge to utilize the available ones adequately are some of the influencing factors.

The provision of adequate pain relief after surgery is an ethical responsibility of healthcare providers and a fundamental right of every patient [20-22]. Its importance has been emphasized during the early stages of recovery $[23,24]$. Effective management of postoperative pain is an essential component in the provision of good quality of care, and it can have a significant effect on patient recovery by improving clinical outcomes, avoiding clinical complications, and finally saving health care resources $[25,26]$. Not only during the postoperative period but adequate pain management plan requires preoperative preparation, up until after discharge to control pain effectively [27]. A preadministered analgesia reduces analgesic requirement [28]. Moreover, the accuracy in pain assessment has a major role in measuring the adequacy of pain management $[4,29]$. Despite such an importance and its impact on the patient, postoperative pain is usually undertreated and suffering from pain continues to be a significant challenge [30-33]. It has also been explained that appropriate postoperative pain management is generally neglected in Eritrea [19]. Lack of skilled professionals and pain management guidelines also contribute to the condition, and pain management is more or less derived from the experience of the staff. In reflection to this, the experience of postoperative pain was found to be high in which the total experience of pain was $82.6 \%$, of whom $43.6 \%$ experienced more than moderate pain. Not only in this study but also in the studies performed using the same questionnaire, postoperative pain was among the most frequently experienced complaints $[17,18,34,35]$. Similar results were also reported in another study from the Netherlands by Kalkman and colleagues. This Dutch study was conducted in a university hospital on 1416 patients undergoing various surgical procedures with the aim of developing a validated prediction rule for the occurrence of early postoperative severe pain in surgical patients, and the incidence of severe pain was found to be $25.8 \%$, measured one hour after surgery [36]. Another similar result was also reported in a study performed in Saudi Arabia. The study was conducted on 199 patients who did surgeries in a university hospital. The same questionnaire (LPPSq) was used, and they found postoperative pain to be the most frequently mentioned complaint [34]. Moreover, in a recent study performed in Mexico, the frequency of pain from moderate to severe level was determined to be $66.3 \%$ in which they concluded that their study represents ineffective pain management practices [30]. Worst pain experience score was also reported among $79 \%$ of the postoperative surgical patients in Ethiopia [37]. In the studies performed by Jlala et al and Calijouw et al., which were conducted with the primary aim of validating the LPPS questionnaire, thirst was another frequently mentioned complaint, unlike in this current study. The other postoperative side effects (back 
TABLE 3: Occurrence of undesirable postoperative anesthesia outcomes (types of surgery versus types of anesthesia).

\begin{tabular}{|c|c|c|c|c|c|c|c|c|c|}
\hline & \multicolumn{3}{|c|}{ General surgery } & \multicolumn{3}{|c|}{ Orthopedic surgery } & \multicolumn{3}{|c|}{ Gyn/Obs surgery } \\
\hline & GA & RA & OR (95\% CI) & GA & RA & OR (95\% CI) & GA & RA & OR $(95 \% \mathrm{CI})$ \\
\hline Posto & 84.66 & 77.55 & $1.60(0.85,3.02)$ & 75.68 & 77.42 & $0.91(0.35,2.37)$ & 92 & 89.74 & $1.31(0.31,5.63)$ \\
\hline Sore throat & 26.38 & 6.12 & $5.49(2.24,13.46)^{* * *}$ & 27.03 & 6.45 & $5.37(1.54,18.67)^{* *}$ & 24 & 10.27 & $2.76(0.82,9.37)$ \\
\hline Back pain & 17.79 & 30.61 & $0.49(0.27,0.88)^{*}$ & 24.32 & 24.19 & $1.01(0.39,2.60)$ & 30 & 56.41 & $0.33(0.14,0.80)^{*}$ \\
\hline Nausea & 49.08 & 35.71 & $1.74(1.04,2.90)^{*}$ & 10.81 & 19.35 & $15,1.70)$ & 60 & 64.1 & $0.84(0.35,2.00)$ \\
\hline Vomiting & 44.79 & 28.57 & $2.03(1.19,3.47)^{*}$ & 16.22 & 16.13 & $1.01(0.33,3.04)$ & 66 & 64.1 & $1.09(0.45,2.62)$ \\
\hline Cold & 44.17 & 40.82 & $1.15(0.70,1.91)$ & 27.03 & 32.26 & $0.79(0.32,1.91)$ & 44 & 58.97 & $0.55(0.23,1.28)$ \\
\hline Hunger & 20.25 & 20.41 & $0.99(0.53,1.85)$ & 2.7 & 14.52 & $0.16(0.02,1.35)$ & 24 & 17.95 & $1.44(0.51,4.10)$ \\
\hline Thirst & 33.13 & 30.61 & $1.12(0.66,1.93)$ & 8.11 & 12.9 & $0.60(0.15,2.40)$ & 36 & 33.33 & $1.13(0.47,2.72)$ \\
\hline Headache & 18.4 & 28.57 & $0.56(0.31,1.02)$ & 27 & 27.42 & $0.98(0.39,2.45)$ & 20 & 38.46 & $0.40(0.16,1.03)$ \\
\hline
\end{tabular}

RA (regional anesthesia)-reference category. ${ }^{*} p<0.05,{ }^{* *} p<0.01,{ }^{* * *} p<0.001$.

pain, headache, hunger, nausea, and vomiting) were less frequently reported with the lowest median score in all the abovementioned studies including this current study. Whereas according to a study performed in Rwanda, which was once again conducted using the same LPPS questionnaire on 145 patients, thirst and hunger were highly reported [35]. The status of postoperative pain was also compared between the two types of anesthesia; however, no significant difference was scored in its occurrence. Unlike in this study, in a study performed by Caljouw et al., 2008, a significant difference was observed in the occurrence of postoperative pain between general anesthesia $(82.1 \%)$ and regional anesthesia (34.3\%).

One of the first unpleasant symptoms in the postoperative period is postoperative nausea and vomiting [1,38]. Despite anesthetic and pharmacological advances, there is no drug that is completely effective in preventing postoperative nausea and vomiting (PONV). They appear frequently, and they can often be complex and can be a significant problem in anesthesia practice. It is also often described as to be the second most common complaint after pain during the postoperative period [38, 39]. Understanding the mechanism and careful assessment of risk factors help in its management $[2,39,40]$. Normally, the occurrence of these unpleasant experiences is seen more commonly related to occur with general anesthesia than when using regional anesthesia $[2,12,13,41]$. Similarly, in this current study, the occurrence of nausea and vomiting was higher among those patients who took general anesthesia. Since postoperative pain is commonly managed with NSAIDs and narcotics are hardly utilized for such purposes, it can be stated that the experience of nausea and vomiting is anesthesia related. As general anesthesia involves manipulation of the airway, it is obvious that the occurrence of sore throat to be higher in those patients who took general anesthesia. The very few sore throat that occurred among those who took regional anesthesia may not be purely associated with the anesthetic procedure. Moreover, the type of anesthesia given to all those who took regional anesthesia was spinal, and thus, the experience of back pain was high among these groups. Hypothermia affects more than $70 \%$ of patients undergoing surgery and anesthesia [13]. The main factors which lead to hypothermia include exposure to cold temperature during the intraoperative period, administration of unwarmed intravenous fluids, and evaporation from within the surgical incision [2, 42]. The deactivation of the thermoregulation center by general anesthesia itself can also lower the core temperature. Moreover, the usage of muscle relaxants during the operation greatly affects the patient's muscles ability to shiver and produce heat thus resulting in temperature drop [42]. This would be more pronounced in settings with limited resources like the operating rooms in the selected Eritrean study settings that otherwise would help to maintain the temperature of the rooms and of that of the patient. Meanwhile, the occurrence of hypothermia among the selected patients was not significant, and neither there was a significant difference in the occurrence of the rest of undesirable anesthesia outcomes such as hunger, thirst, and headache. Generally, postoperative undesirable events still persist despite the medical advances. There is a need to improve the treatment of these events by administering effective methods through an organized and systematized care, which is consistent with each setting. Such an approach would facilitate patient's recovery and shortens the hospital stay and will have a positive impact on patient's outcome and wellbeing [30].

Preoperative assessment is an important component of anesthesia [43]. Preparing a patient for anesthesia requires an understanding of the patient's preoperative status, the nature of the surgery, the anesthetic techniques required for surgery, and the risks that a particular patient may face during this time [44]. Problems with adequacy of preoperative assessment in the study settings, which was mentioned in a previous publication from this study [19], may also contribute to the increment in the prevalence of these undesirable outcomes. Patients should be provided with adequate information of the possible postoperative outcomes during the preoperative assessment, and depending on the results of this study, techniques of managing postoperative pain should be considered and discussed starting from the preoperative period. Postoperative care should be delivered by a multidisciplinary team that includes the anesthetist to gain a better outcome. It is very important to scale the level of pain so as to provide a management that corresponds with the level of severity. The type of surgery and type of anesthesia should also be considered. Narcotic agents alone can be given in conjunction with regional anesthesia, and their effect may also be extended by 
continuing the treatment with NSAIDs. This would reduce the undesirable effect from the narcotics and enhance postoperative pain management.

4.1. Limitations of the Study. Some of the exclusion criteria may have impacted the results of the study and the fact that the participants were made to be interviewed before their discharge, and the dependence of care might have retrained them from speaking their mind.

\section{Conclusion}

Postoperative pain was the most predominant undesirable anesthesia outcome. This gives a signal for the study settings to consider the involvement of anesthetists or dedicated pain nurses in the management of postoperative pain. This would enhance the proper management of postoperative pain through the development and implementation of specific pain management modalities. Local guidelines should be prepared in these settings to use for the treatment of acute postoperative pain. Moreover, measuring anesthetic outcomes in a reliable and valid manner would also be important for improving the standards of anesthetic care and delivering the quality of anesthesia in these settings. It has also been reported that adequate information is not provided to patients regarding their postoperative experience. Therefore, it is required for the study settings to make efforts on providing consistent preoperative information regarding choices for anesthesia, the risks and benefits of the drugs, postoperative analgesia, prevention and treatment of other outcomes, and obtaining maximum efficacy from the administered treatments. Further research is also needed to determine on how to develop further management strategies of these undesirable events.

\section{Abbreviations}

$\begin{array}{ll}\text { CI: } & \text { Confidence interval } \\ \text { ENT: } & \text { Ear, nose, and throat } \\ \text { GA: } & \text { General anesthesia } \\ \text { Gyn/ } & \text { Gynecology and Obstetrics } \\ \text { Obs: } & \\ \text { LPPSq: } & \text { Leiden Perioperative care Patient Satisfaction } \\ & \text { questionnaire } \\ \text { NSAIDs: } & \text { Nonsteroidal anti-inflammatory drugs } \\ \text { OR: } & \text { Odds ratio } \\ \text { POD: } & \text { Postoperative day } \\ \text { PONV: } & \text { Postoperative nausea and vomiting } \\ \text { RA: } & \text { Regional anesthesia } \\ \text { SD: } & \text { Standard deviation } \\ \text { SPSS: } & \text { Statistical Package for the Social Sciences } \\ \text { UAO: } & \text { Undesirable anesthesia outcomes. }\end{array}$

\section{Data Availability}

The datasets generated and/or analyzed during the current study are available from the corresponding author upon request.

\section{Ethical Approval}

Ethical clearance and approval were obtained from the Ethical and Scientific Committee of the Asmara College of Health Sciences and from the Health Research Proposal Review and Ethical Committee of the Ministry of Health with a reference number of $11 / 10 / 17$. The members of the ethics committee were namely Dr. Brhane Debru, Mr. Salih Gemam, and Mr. Mehari Weldu. Permission was also secured from both hospitals.

\section{Consent}

After explaining the purpose of the study and assurance of confidentiality and anonymity, informed written consent was obtained from each participant, and the interview was conducted. Name and other identifying information are not included in the study.

\section{Disclosure}

The funder had no role in the design of the project, collection, analysis, and interpretation of data, or in writing and publication of the manuscript.

\section{Conflicts of Interest}

The authors declare that there are no conflicts of interest.

\section{Acknowledgments}

The authors would like to thank the National Commission for Higher Education (NCHE) for funding the research.

\section{References}

[1] D. Malczyk et al., "Are there any unwanted anesthesia symptoms in patients undergoing anesthesia?" Journal of Education, Health and Sport, vol. 8, no. 9, pp. 77-89, 2018.

[2] L. Paavolainen and J. Wallstedt, Post-operative Complications of General Anesthesia: A Recorded Video Presentation, Jyväskylä University of Applied, Jyväskylä, Finland, 2016.

[3] D. Darlene Falco and C. D. N. Rutledge, "Patient satisfaction with anesthesia care: what do we know?" AANA Journal, vol. 85, no. 4, p. 287, 2017.

[4] M. S. Abdalrahim, Postoperative Pain Assessment and Management: The Effects of an Educational Program on Jordanian Nurses' Practice, Knowledge, and Attitudes, Institute of Health and Care Sciences, Gothenburg, Sweden, 2009.

[5] D. Fletcher, C. Fermanian, A. Mardaye, and P. Aegerter, "A patient-based national survey on postoperative pain management in France reveals significant achievements and persistent challenges," Pain, vol. 137, no. 2, pp. 441-451, 2008.

[6] P. Dumolard, M. Gök, and N. Le, Nurses' Responsibilities in Postoperative Pain Management Following Total Hip Arthroplasty, JAMK University of Applied Sciences, Jyväskylä, Finland, 2017.

[7] D. Milutinovic, V. Milovanovic, M. Pjevic, M. MartinovCvejin, and T. Cigic, "Assessment of quality of care in acute postoperative pain management," Vojnosanitetski Pregled, vol. 66, no. 2, pp. 156-162, 2009. 
[8] G. Savoia, D Alampi, B Amantea et al., "Postoperative pain treatment SIAARTI Recommendations 2010. Short version," Minerva Anestesiologica, vol. 76, no. 8, pp. 657-667, 2010.

[9] T. Sada Ovalle, E. Delgado Hernández, and A. Castellanos Olivares, "Prevalencia del dolor postoperatorio en cirugía electiva de pacientes del hospital de especialidades del Centro Médico Nacional Siglo XXI IMSS," Revista de la Sociedad Española del Dolor, vol. 18, no. 2, pp. 91-97, 2011.

[10] J. Muñoz, Manual de dolor agudo postoperatorio, Ergon, Jackson, MI, USA, 2010.

[11] L. Fadaeizadeh, H. Emami, and K. Samiei, "Comparison of visual analogue scale and faces rating scale in measuring acute postoperative pain," Archives of Iranian Medicine, vol. 12, 2009.

[12] T. J. Gan, "Risk factors for postoperative nausea and vomiting," Anesthesia \& Analgesia, vol. 102, no. 6, pp. 1884-1898, 2006.

[13] T. Belayneh, A. Gebeyehu, and Z. Abdissa, "Post-operative hypothermia in surgical patients at university of gondar hospital, Ethiopia," Journal of Anesthesia and Clinical Research, vol. 5, no. 461, p. 2, 2014.

[14] A. R. Aitkenhead, "Injuries associated with anaesthesia. A global perspective," British Journal of Anaesthesia, vol. 95, no. 1, pp. 95-109, 2005.

[15] S. Naidu and P. Gopalan, "The informed consent process for anaesthesia: perspectives of elective surgical patients at Inkosi Albert Luthuli Central Hospital, Durban, South Africa," Southern African Journal of Anaesthesia and Analgesia, vol. 22, no. 2, pp. 60-64, 2016.

[16] S. F. Barnett, R. K. Alagar, M. P. W. Grocott, S. Giannaris, J. R. Dick, and S. R. Moonesinghe, "Patient-satisfaction measures in anesthesia," Anesthesiology, vol. 119, no. 2, pp. 452-478, 2013.

[17] M. A. A. Caljouw, M. Van Beuzekom, and F. Boer, "Patient's satisfaction with perioperative care: development, validation, and application of a questionnaire," British Journal of Anaesthesia, vol. 100, no. 5, pp. 637-644, 2008.

[18] H. Jlala, A. C. Monique, M. B. Nigel, and G. H. Jonathan, "Patient satisfaction with perioperative care among patients having orthopedic surgery in a university hospital," Local and Regional Anesthesia, vol. 3, p. 49, 2010.

[19] Y. M. Andemeskel, E. Traudl, G. Ghidey, and H. T. Eyasu, "Patient satisfaction with peri-operative anesthesia care and associated factors at two National Referral Hospitals: a cross sectional study in Eritrea," BMC Health Services Research, vol. 19, no. 1, p. 669, 2019.

[20] J. Tuchscherer, W. P. McKay, and T. Twagirumugabe, "Lowdose subcutaneous ketamine for postoperative pain management in Rwanda: a dose-finding study," Canadian Journal of Anesthesia/Journal Canadien D’anesthésie, vol. 64, no. 9, pp. 928-934, 2017.

[21] T. J. Brennan and H. Kehlet, "Preventive analgesia to reduce wound hyperalgesia and persistent postsurgical pain," $A n$ esthesiology, vol. 103, no. 4, p. 681, 2005.

[22] M. Size, O. A. Soyannwo, and D. M. Justins, "Pain management in developing countries," Anaesthesia, vol. 62, no. s1, pp. 38-43, 2007.

[23] M. Joelsson, L.-E. Olsson, and E. Jakobsson, "Patients' experience of pain and pain relief following hip replacement surgery," Journal of Clinical Nursing, vol. 19, no. 19-20, pp. 2832-2838, 2010.

[24] L. Frenette, "The acute pain service," Critical Care Clinics, vol. 15, no. 1, pp. 143-150, 1999.
[25] J. L. Apfelbaum, C. Chen, S. S. Mehta, and a. T. J. Gan, "Postoperative pain experience: results from a national survey suggest postoperative pain continues to be undermanaged," Anesthesia \& Analgesia, vol. 97, no. 2, pp. 534-540, 2003.

[26] S. J. Dolin, J. N. Cashman, and J. M. Bland, "Effectiveness of acute postoperative pain management: I. Evidence from published data," British Journal of Anaesthesia, vol. 89, no. 3, pp. 409-423, 2002.

[27] R. Chou, D. B. Gordon, O. A. de Leon-Casasola et al., "Management of Postoperative Pain: a clinical practice guideline from the American pain society, the American Society of Regional Anesthesia and Pain Medicine, and the American Society of Anesthesiologists' committee on regional anesthesia, executive committee, and administrative council," The Journal of Pain, vol. 17, no. 2, pp. 131-157, 2016.

[28] V. Garimella and C. Cellini, "Postoperative pain control," Clinics in Colon and Rectal Surgery, vol. 26, no. 3, pp. 191-196, 2013.

[29] M. W. Stomberg and H. Haljamäe, "Postoperative pain management-impact of quality assurance and audit documentation on clinical outcome," Current Anaesthesia \& Critical Care, vol. 14, no. 5-6, pp. 217-221, 2003.

[30] P. E. García-Ramírez et al., "Postoperative pain: frequency and management characterization," Revista colombiana de anestesiologia, vol. 46, no. 2, pp. 93-97, 2018.

[31] E. Manias, T. Bucknall, and M. Botti, "Nurses' strategies for managing pain in the postoperative setting," Pain Management Nursing, vol. 6, no. 1, pp. 18-29, 2005.

[32] M. Helfand and M. Freeman, "Assessment and management of acute pain in adult medical inpatients: a systematic review," Pain Medicine, vol. 10, no. 7, pp. 1183-1199, 2009.

[33] M. Botti, T. Bucknall, and E. Manias, "The problem of postoperative pain: issues for future research," International Journal of Nursing Practice, vol. 10, no. 6, pp. 257-263, 2004.

[34] G. A. El-Nasser and N. Mohamed, "Patient satisfaction with preoperative care and its relationship with patient characteristics," The Medical Journal of Cairo University, vol. 81, no. 2, 2013.

[35] L. Ingabire, Patients Satisfaction with Perioperative Care at Oshen Fing Feisal Hospital, University of Rwanda, Kigali, Rwanda, 2017.

[36] J. C. Kalkman, K. Visser, J. Moen, J. G. Bonsel, E. D. Grobbee, and M. K. G. Moons, "Preoperative prediction of severe postoperative pain," Pain, vol. 105, no. 3, pp. 415-423, 2003.

[37] T. E. Woldehaimanot, T. C. Eshetie, and M. W. Kerie, "Postoperative pain management among surgically treated patients in an Ethiopian hospital," PloS One, vol. 9, no. 7, Article ID e102835, 2014.

[38] T. J. Gan, F. Sloan, G. de L Dear, H. E. El-Moalem, and D. A. Lubarsky, "How much are patients willing to pay to avoid postoperative nausea and vomiting?" Anesthesia and Analgesia, vol. 92, no. 2, pp. 393-400, 2001.

[39] S. I. Shaikh, D. Nagarekha, H. Ganapati, and M. Marutheesh, "Postoperative nausea and vomiting: a simple yet complex problem," Anesthesia, Essays and Researches, vol. 10, no. 3, p. 388, 2016.

[40] A. Lee and M. E. Lum, "Measuring anaesthetic outcomes," Anaesthesia and Intensive Care, vol. 24, no. 6, pp. 685-693, 1996.

[41] M. Stadler, F. Bardiau, L. Seidel, A. Albert, and J. G. Boogaerts, "Difference in risk factors for postoperative nausea and vomiting," Anesthesiology, vol. 98, no. 1, pp. 46-52, 2003. 
[42] D. I. Sessler, "Temperature monitoring and perioperative thermoregulation," Anesthesiology, vol. 109, no. 2, p. 318, 2008.

[43] S. M. Yentis, A. J. Hartle, I. R. Barker et al., "AAGBI: consent for anaesthesia 2017," Anaesthesia, vol. 72, no. 1, pp. 93-105, 2017.

[44] G. Vyhunthan and N. de Silva, "Audit to evaluate preoperative visit to patient by anaesthetist," Sri Lankan Journal of Anaesthesiology, vol. 20, no. 2, 2012. 